\title{
Теория упругости
}

УДК 539.3

DOI: $10.22363 / 1815-5235-2017-6-16-24$

\section{АНАЛИЗ НАПРЯЖЕННОГО СОСТОЯНИЯ ТРЕХСЛОЙНОГО АНИЗОТРОПНОГО ОСНОВАНИЯ}

С.Г. КУДРЯВЦЕВ, канд. техн. наук, доиент Ю.М. БУЛДАКОВА, ст. преподаватель

ФГБОУ ВО «Поволюсский государственный технологический университет» 424000, Республика Марий Эл, г. Йошкар-Ола, пл. Ленина, дом 3

KudryavcevSG@volgatech.net

Представлены результаты расчетов на основе уравнений плоской задачи теории упругости по определению напряжений в трехслойном анизотропном основании под действием статической нормальной поверхностной нагрузки. Рассматривается вариант полного сиепления между слоями.

Функиии напряжений в каждом слое записываются через нормальные и касательные усилия, возникающце на линии контакта полосы и полуплоскости. Контактные усилия между полосой и полуплоскостью определяются из системь двух уравнений и условий на поверхности основания. В результате функиии напряжений в каждом слое записываются через поверхностную нагрузку в виде несобственных интегралов.

Проводится анализ напряженного состояния по модели трехслойного изотропного основания в зависимости от упругих характеристик материала слоев. Показано влияние анизотропии материала слоев в трехслойном грунтовом массиве.

КЛЮЧЕВЫЕ СЛОВА: напряжение, анизотропия, упругость, полоса, полуплоскость.

Теоретические и экспериментальные исследования напряженно- деформированного состояния в многослойных основаниях имеют практическое приложение в механике грунтов [1], при расчете дорожных и аэродромных покрытий [2]. Из ранних исследований, связанных с расчетом слоистых изотропных оснований под действием поверхностной нагрузки, отметим работы [3-5]. Данный вопрос рассматривался также в статьях [6-10]. Применение изотропной модели при расчете многослойных оснований, когда материал слоев имеет ярко выраженную анизотропию приводит, как показано, например, в публикациях [1114], к искажению действительной картины работы основания.

В работе представлены результаты расчетов по определению напряжений в трехслойном анизотропном основании при условии полного сцепления между слоями, под действием статической поверхностной нагрузки, и проводится их сопоставление с расчетом по модели трехслойного изотропного основания.

Рассмотрим трехкомпонентную упругую среду, состоящую из двух полос с плоскопараллельными границами бесконечной длины и полуплоскости (рис.1). Материал каждого слоя анизотропный с заданными упругими характеристиками. Для полос упругие характеристики материала, высота, напряжения, перемещения обозначены нижним индексом $k(k=1,2)$. Нумерация принята снизу вверх. Перемещения, напряжения, упругие характеристики материала полуплоскости обозначим чертой сверху. Каждый слой отнесем к местной декартовой системе координат. Оси абсцисс всех систем координат сонаправлены. Оси ординат совпадают и направлены вглубь слоёв перпендикулярно границе раздела. Положительные направления напряжений и перемещений для полос и полуплоскости показаны на рисунке.

При полном сцеплении между слоями на линии соединения полосы и полуплоскости выполняются следующие условия контакта: 


$$
\begin{gathered}
\sigma_{y 1}\left(x_{1}, y_{1}=0\right)=\bar{\sigma}_{y}(\bar{x}, \bar{y}=0) ; \tau_{x y 1}\left(x_{1}, y_{1}=0\right)=\bar{\tau}_{x y}(\bar{x}, \bar{y}=0) ; \\
u_{1}\left(x_{1}, y_{1}=0\right)=\bar{u}(\bar{x}, \bar{y}=0) ;-v_{1}\left(x_{1}, y_{1}=0\right)=\bar{v}(\bar{x}, \bar{y}=0) ;
\end{gathered}
$$

на линии взаимодействия полос условия имеют вид:

$$
\begin{gathered}
\sigma_{y 1}\left(x_{1}, y_{1}=h_{1}\right)=\sigma_{y 2}\left(x_{2}, y_{2}=0\right) ; \tau_{x y 1}\left(x_{1}, y_{1}=h_{1}\right)=\tau_{x y 2}\left(x_{2}, y_{2}=0\right) ; \\
u_{1}\left(x_{1}, y_{1}=h_{1}\right)=u_{2}\left(x_{2}, y_{2}=0\right) ; \quad v_{1}\left(x_{1}, y_{1}=h_{1}\right)=v_{2}\left(x_{2}, y_{2}=0\right) .
\end{gathered}
$$

На поверхности основания заданы граничные условия первого рода:

$$
\sigma_{y_{2}}\left(x_{2}, y_{2}=h_{2}\right)=q(x) ; \tau_{x y 2}\left(x_{2}, y_{2}=h_{2}\right)=\tau(x) .
$$

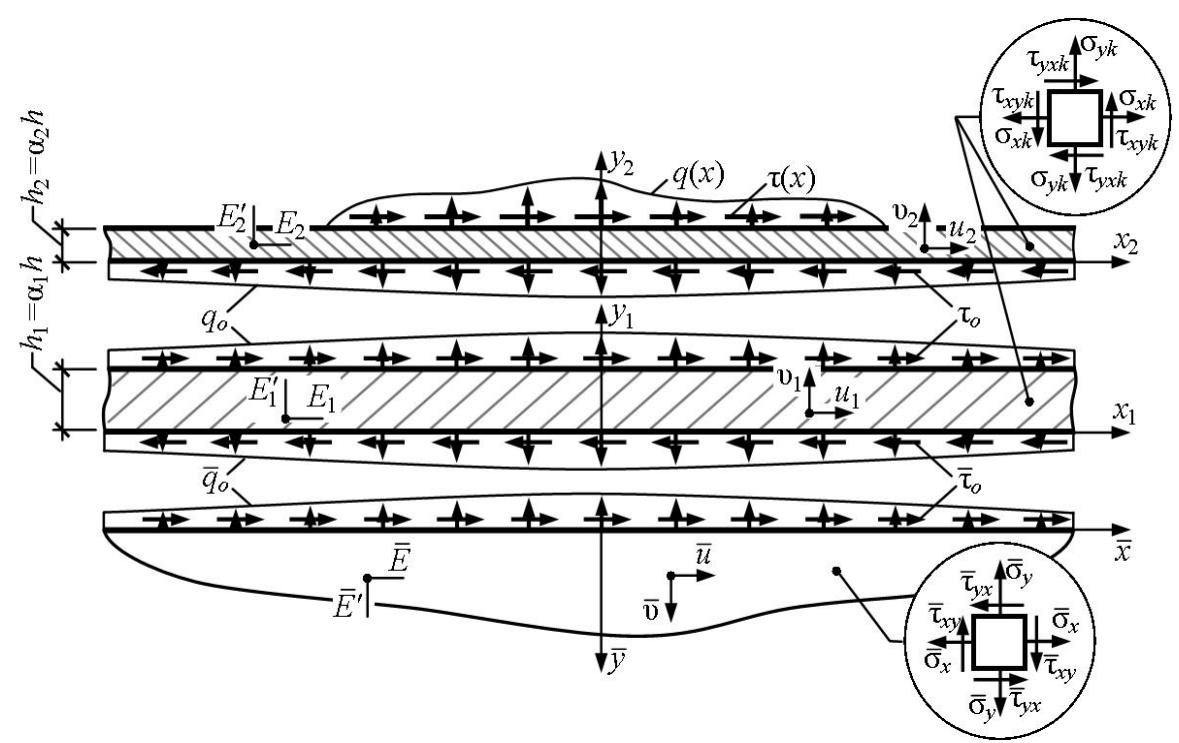

Рис.1. Схема взаимодействия слоев основания

Задача решалась на основе метода, изложенного в [15]. Используя уравнения для определения напряжений и перемещений в полосе и полуплоскости, приведенные в [15-16], и условия (1)-(2), функции перемещений и напряжений в каждом слое запишем через нормальные и касательные усилия, возникающие на линии контакта полосы и полуплоскости. Полагая, что существует интегральное преобразование Фурье от функций $q(x)$ и $\tau(x)$, выразим из условий (3) контактные усилия между полосой и полуплоскостью через поверхностную нагрузку. В результате функции перемещений и напряжений в каждом слое будут записаны через поверхностную нагрузку в виде несобственных интегралов, которые, ввиду их громоздкой структуры, не приводятся.

Приведем результаты расчетов по определению напряжений на конкретном примере. Пусть на участке длиной $2 a$ действует перпендикулярно плоскости основания распределенная нагрузка постоянной интенсивности $q$ (рис.2). Полагаем, что слои выполнены из разных изотропных материалов (коэффициент Пуассона $\left.\bar{v}=v_{k}=0,25\right)$, высота полос одинакова $\left(\alpha_{k}=1\right)$, отношение $h / a=2$. Расчеты выполнены для плоского деформированного состояния.

При проведении расчетов вводим безразмерные параметры для полос:

$$
\tilde{\sigma}_{y k}=\frac{\sigma_{y k} \pi}{q}, \tilde{\sigma}_{x k}=\frac{\sigma_{x k} \pi}{q}, \tilde{\tau}_{x y k}=\frac{\tau_{x y k} \pi}{q}, \tilde{x}_{k}=\frac{x_{k}}{h}, \tilde{y}_{k}=\frac{y_{k}}{h}, \alpha_{k}=\frac{h_{k}}{h},
$$

и полуплоскости:

$$
\widetilde{\bar{\sigma}}_{y}=\frac{\bar{\sigma}_{y} \pi}{q}, \widetilde{\bar{\sigma}}_{x}=\frac{\bar{\sigma}_{x} \pi}{q}, \widetilde{\bar{\tau}}_{x y k}=\frac{\bar{\tau}_{x y} \pi}{q}, \widetilde{\bar{x}}=\frac{\bar{x}}{h}, \widetilde{\bar{y}}=\frac{\bar{y}}{h},
$$




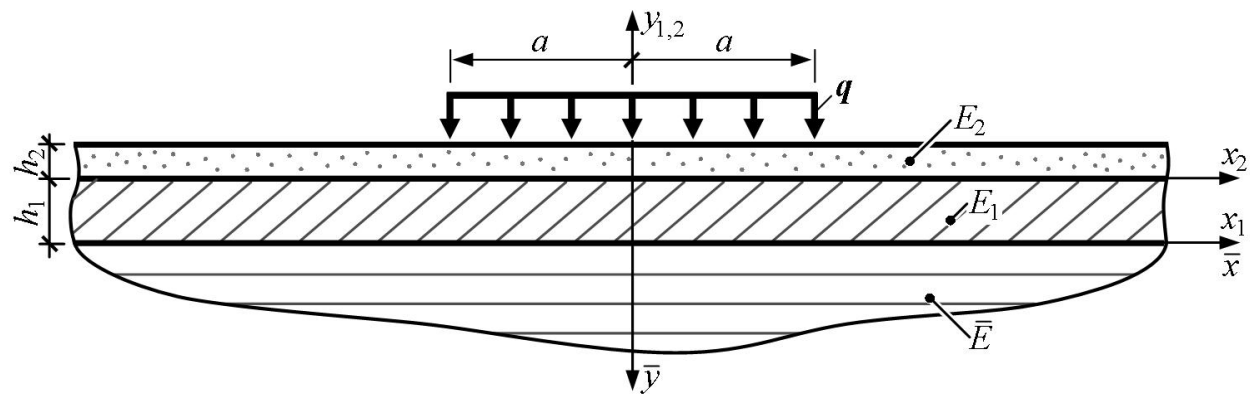

Рис. 2. Схема нагружения трехслойного изотропного основания

Параметры напряжений для полос и полуплоскости, безразмерные параметры координат далее будем обозначать одинаково: $\widetilde{\sigma}_{x}, \widetilde{\sigma}_{y}, \widetilde{\tau}_{x y}, \widetilde{x}$.

Сравнение данных в таблице 1 , где приведены максимальные значения $\widetilde{\sigma}_{y}$, показывает, что увеличение модуля упругости в вышележащих слоях приводит к уменьшению $\left|\tilde{\sigma}_{y}^{\max }\right|$ во всех слоях. Для варианта однородного изотропного основания $\left(E_{1} / \bar{E}=E_{2} / E_{1}=1\right)$ значения совпадают с данными [1].

Таблица 1. Значения $\left|\widetilde{\sigma}_{y}^{\max }\right|$ в зависимости от отномения модулей упругости материала слоев и параметра $\widetilde{y}_{k}(\widetilde{\bar{y}})$

\begin{tabular}{|c|c|c|c|c|c|c|c|}
\hline \multirow{2}{*}{\multicolumn{2}{|c|}{$\frac{E_{1} / \bar{E}}{E_{2} / E_{1}}$}} & \multicolumn{3}{|c|}{1} & \multicolumn{3}{|c|}{2} \\
\hline & & 1 & 2 & 5 & 1 & 2 & 5 \\
\hline \multirow[b]{2}{*}{$\tilde{y}_{2}$} & 0,5 & 2,57 & 2,47 & 2,31 & 2,55 & 2,45 & 2,28 \\
\hline & 0,0 & 1,73 & \multirow{2}{*}{1,55} & \multirow{2}{*}{1,27} & \multirow{2}{*}{1,67} & \multirow{2}{*}{1,48} & 1,19 \\
\hline \multirow{2}{*}{$\tilde{y}_{1}$} & $\begin{array}{l}1,0 \\
0,5\end{array}$ & 1,24 & & & & & 0,853 \\
\hline & 0,0 & \multirow{2}{*}{0,961} & \multirow{2}{*}{0,880} & \multirow{2}{*}{0,769} & \multirow{2}{*}{0,847} & \multirow{2}{*}{0,768} & \multirow{2}{*}{0,666} \\
\hline \multirow{2}{*}{$\widetilde{\bar{y}}$} & 0,0 & & & & & & \\
\hline & 1,0 & 0,655 & 0,611 & 0,553 & 0,588 & 0,543 & 0,487 \\
\hline
\end{tabular}

Результаты вычислений параметра $\left|\tau_{x y}^{\max }\right|$ в горизонтальных сечениях основания, в зависимости от отношения модулей упругости материала слоев и параметра $\tilde{y}_{k}(\tilde{\bar{y}})$, показаны в таблице 2 . В числителе указана максимальная величина $\tilde{\tau}_{x y}$, в знаменателе - координата $|\widetilde{x}|$, в которой возникает $\left|\tilde{\tau}_{x y}^{\max }\right|$. Из сопоставления данных следует, что при увеличении модуля упругости материала верхней полосы значение $\left|\widetilde{\tau}_{x y}^{\max }\right|$ в этой полосе возрастает, а в нижних слоях уменьшается. При увеличении отношения $E_{1} / \bar{E}$ значение $\left|\tilde{\tau}_{x y}^{\max }\right|$ возрастает в обеих полосах и уменьшается в полуплоскости.

На рис. 3,a представлены графики изменения параметра $\widetilde{\sigma}_{x}$ на нижней плоскости верхней полосы, на рис.3б - на верхней плоскости нижней полосы и указаны значения $\widetilde{\sigma}_{x}^{\max }$. Кривая 1 соответствует случаю, когда модули упругости материала слоев одинаковы, кривая 2 построена при отношении $E_{1} / \bar{E}=E_{2} / E_{1}=2,3$ - для варианта $E_{1} / \bar{E}=2, E_{2} / E_{1}=5$. Видно, что при одном и том же значении координаты $\tilde{x}$ на линии взаимодействия, когда материал полос имеет разные модули упругости, параметры $\widetilde{\sigma}_{x}$ отличаются по величине, а при некоторых значениях $\tilde{x}$ и по знаку. 
Таблица 2. Значение $\left|\tilde{\tau}_{x y} \max \right|$ в зависимости от отношения модулей упругости материала слоев и параметра $\tilde{y}_{k}(\widetilde{\bar{y}})$

\begin{tabular}{|c|c|c|c|c|c|c|c|}
\hline \multirow{2}{*}{\multicolumn{2}{|c|}{$\begin{array}{l}E_{1} / \bar{E} \\
E_{2} / E_{1}\end{array}$}} & \multicolumn{3}{|c|}{1} & \multicolumn{3}{|c|}{2} \\
\hline & & 1 & 2 & 5 & 1 & 2 & 5 \\
\hline \multirow{3}{*}{$\tilde{y}_{2}$} & & 0,806 & 0,930 & 1,14 & 0,840 & 0,972 & 1,19 \\
\hline & 0,3 & 0,538 & 0,551 & 0,575 & 0,544 & 0,559 & 0,584 \\
\hline & 0,0 & 0,552 & 0,512 & 0,410 & 0,609 & 0,572 & 0,462 \\
\hline \multirow{4}{*}{$\widetilde{y}_{1}$} & 1,0 & 0,717 & 0,765 & 0,871 & 0,746 & 0,803 & 0,925 \\
\hline & 0.5 & 0,401 & 0,369 & 0,311 & 0,451 & 0,412 & 0,342 \\
\hline & & 0,960 & 1,05 & 1,21 & 0,99 & 1,08 & 1,26 \\
\hline & 0,0 & 0,310 & 0,289 & 0,253 & 0,281 & 0,259 & 0,226 \\
\hline \multirow[b]{2}{*}{$\widetilde{\bar{y}}$} & 0,0 & 1,23 & 1,33 & 1,54 & 1,33 & 1,45 & 1,69 \\
\hline & 1,0 & $\begin{array}{c}0,212 \\
1,79\end{array}$ & $\begin{array}{c}0,201 \\
1,91\end{array}$ & $\frac{0,185}{2,13}$ & $\begin{array}{c}0,193 \\
1,95\end{array}$ & $\frac{0,181}{2,10}$ & $\frac{0,166}{2,38}$ \\
\hline
\end{tabular}

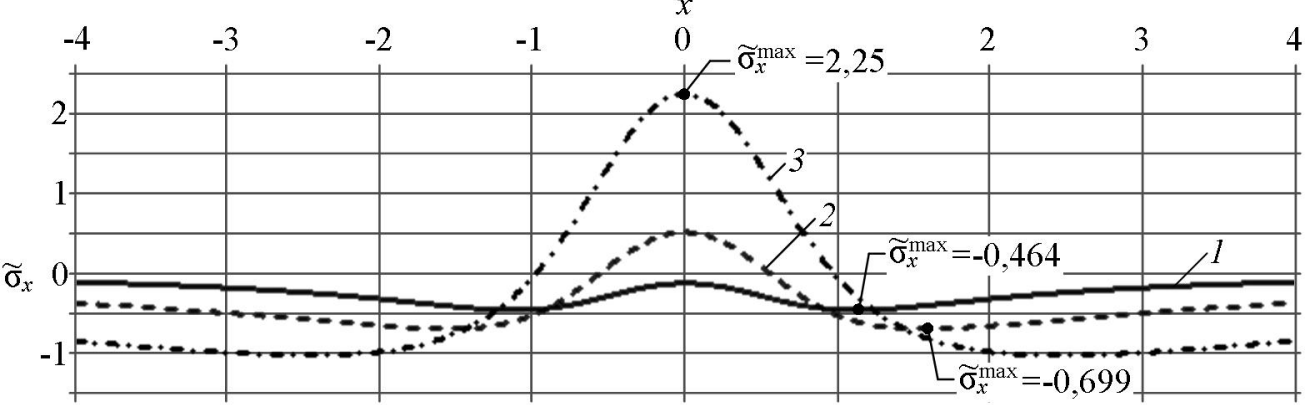

a. Изменение параметра $\widetilde{\sigma}_{x}$ на нижней плоскости верхней полосы

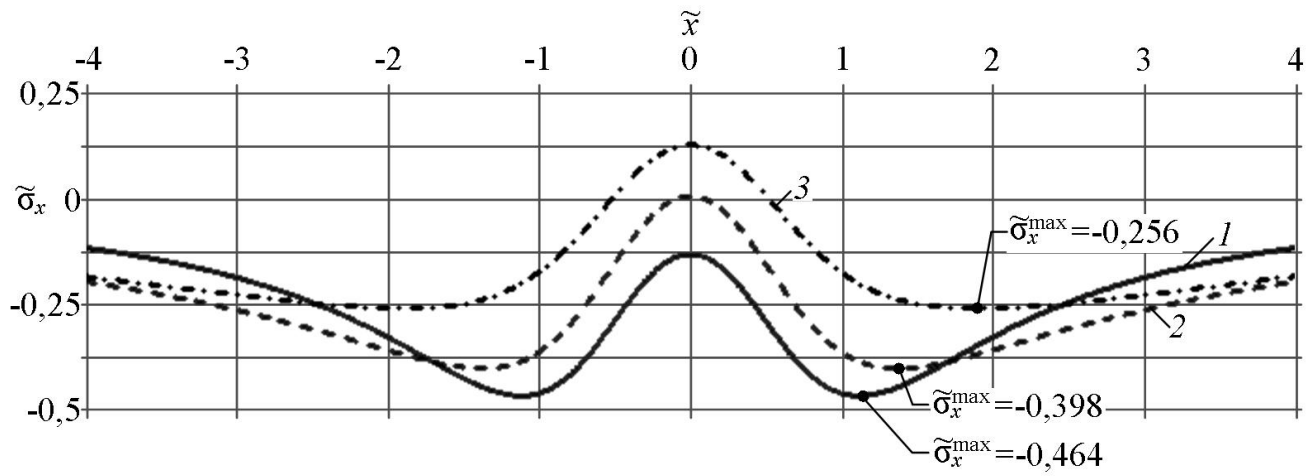

б. Изменение параметра $\widetilde{\sigma}_{x}$ на верхней плоскости нижней полосы

Рис. 3

В таблице 3 в числителе, в зависимости от величины $\tilde{y}_{k}(\widetilde{\bar{y}})$ и отношения модулей упругости материала слоев, показаны максимальные значения параметра $\widetilde{\sigma}_{x}$, в знаменателе - координата $|\widetilde{x}|$, где возникает $\widetilde{\sigma}_{x}^{\max }$.

Рассмотрим влияние анизотропии грунта на примере основания, сложенного из намывного песчаного грунта, супесей и глинистых отложений (рис. 4). Мощность верхних слоев основания одинакова $\left(h_{k}=h\right)$. Вертикальная распределенная нагрузка постоянной интенсивности $q$ действует на участке длиной $2 a=h$ симметрично относительно оси ординат. Для грунтов упругие постоянные приняты согласно [12]: песчаный намывной грунт $-E_{2}=23,0 M П a$, 
$E_{2}^{\prime}=16,0 M \Pi a^{*}, \quad v_{2}^{\prime}=0,30^{*}, \quad G_{2}^{\prime}=7,0 M \Pi a ;$ супесь текучая $-E_{1}=19,6 M \Pi a$, $E_{1}^{\prime}=18,4 M \Pi a^{*}, \quad v_{1}^{\prime}=0,30^{*}, \quad G_{1}^{\prime}=7,1 M \Pi a ;$ слоистые глинистые отложения $\bar{E}=67,5 M \Pi a, \bar{E}^{\prime}=17,0 M \Pi a^{*}, \bar{v}^{\prime}=0,04^{*}$.

Таблица 3. Значения $\widetilde{\sigma}_{x}^{\max } \cdot 10$ в зависимости от отношения модулей упругости материала слоев и параметра $\tilde{y}_{k}(\widetilde{\bar{y}})$

\begin{tabular}{|c|c|c|c|c|c|c|c|}
\hline \multicolumn{2}{|c|}{$E_{1} / \bar{E}$} & \multicolumn{5}{c|}{1} & \multicolumn{2}{c|}{2} \\
\hline \multicolumn{2}{|c|}{$E_{2} / E_{1}$} & 1 & 2 & 5 & 1 & 2 & 5 \\
\hline \multirow{3}{*}{$\tilde{y}_{2}$} & 0,5 & $\frac{-7,85}{0,708}$ & $\frac{-9,31}{0,707}$ & $\frac{-11,4}{0,718}$ & $\frac{-9,95}{0,695}$ & $\frac{-12,0}{0,695}$ & $\frac{-14,6}{0,707}$ \\
\cline { 2 - 9 } & 0,0 & $\frac{-4,64}{1,12}$ & $\frac{-6,45}{1,48}$ & $\frac{19,7}{0,00}$ & $\frac{-5,36}{1,10}$ & $\frac{-6,99}{1,56}$ & $\frac{22,5}{0,00}$ \\
\hline \multirow{5}{*}{$\tilde{y}_{1}$} & 1,0 & $\frac{-4,64}{1,12}$ & $\frac{-3,79}{1,29}$ & $\frac{-2,69}{1,56}$ & $\frac{-5,36}{1,10}$ & $\frac{-3,98}{1,37}$ & $\frac{-2,56}{1,88}$ \\
\cline { 2 - 9 } & 0,5 & $\frac{-3,22}{1,58}$ & $\frac{-2,71}{1,80}$ & $\frac{-2,03}{2,18}$ & $\frac{-3,40}{2,00}$ & $\frac{-2,80}{2,50}$ & $\frac{2,11}{0,00}$ \\
\cline { 2 - 9 } & 0,0 & $\frac{-2,45}{2,06}$ & $\frac{-2,12}{2,31}$ & $\frac{-1,65}{2,74}$ & $\frac{3,60}{0,00}$ & $\frac{3,85}{0,00}$ & $\frac{3,81}{0,00}$ \\
\hline \multirow{2}{*}{$\tilde{y}^{*}$} & 0,0 & $\frac{-2,45}{2,06}$ & $\frac{-2,12}{2,31}$ & $\frac{-1,65}{2,74}$ & $\frac{-1,97}{2,40}$ & $\frac{-1,68}{2,76}$ & $\frac{-1,30}{3,35}$ \\
\cline { 2 - 9 } & 1,0 & $\frac{-1,65}{3,04}$ & $\frac{-1,48}{3,33}$ & $\frac{-1,22}{3,80}$ & $\frac{-1,38}{3,48}$ & $\frac{-1,21}{3,88}$ & $\frac{-0,986}{4,49}$ \\
\hline
\end{tabular}

Значение модуля сдвига для нижнего слоя $\bar{G}^{\prime}=12,76 M П а$ вычислено по формуле [12]. Характеристики, принятые для расчета по модели трехслойного изотропного основания, отмечены (*).

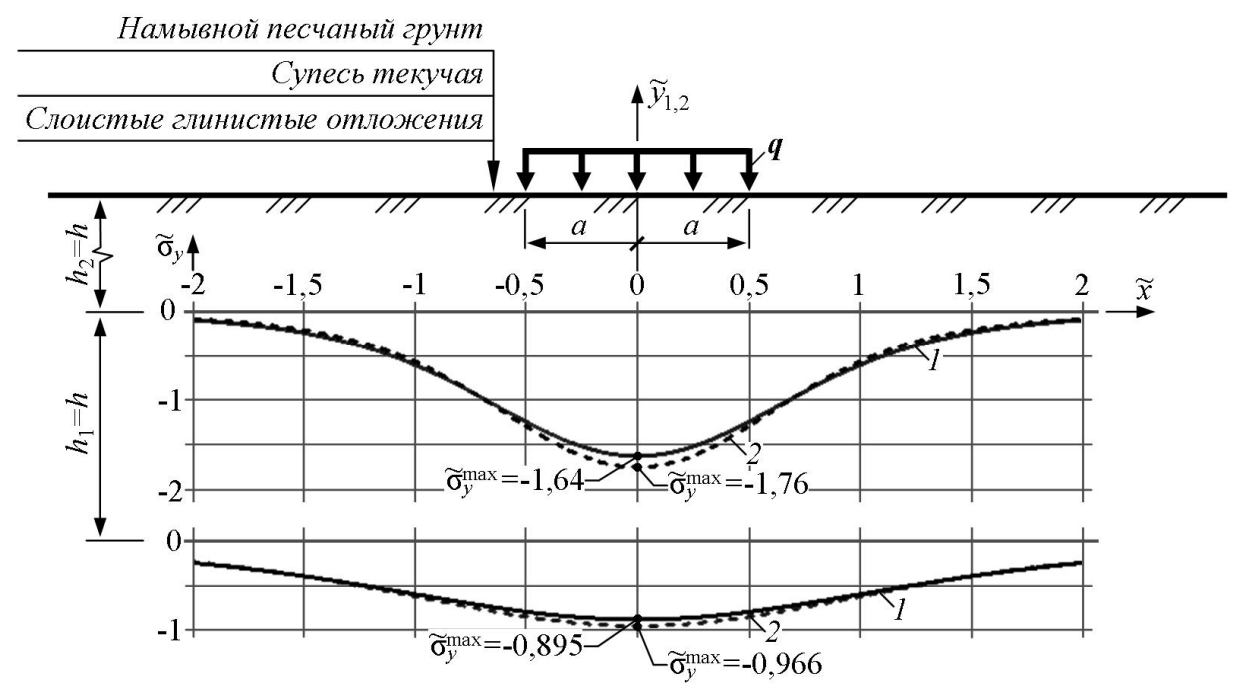

Рис. 4. Изменение параметра $\widetilde{\sigma}_{y}$ на линии контакта слоев

Графики изменения параметров $\tilde{\sigma}_{y}, \tilde{\tau}_{x y}$ на линии контакта полос и на линии взаимодействия полосы и полуплоскости показаны на рисунках 4 и 5 . Цифрой 1 обозначены кривые, построенные по модели трехслойного анизотропного основания, цифрой 2 - трехслойного изотропного. Характер кривых при расчете по анизотропной и изотропной моделям совпадает. Из сравнения кривых на рис.4 видно, что значение $\widetilde{\sigma}_{y}^{\max }$ (при заданных упругих характеристиках материала слоев) больше для трехслойного изотропного основания. 
Анализ кривых на рис.5 показывает, что для рассматриваемого трехслойного основания наличие анизотропии не оказывает значительного влияния на величину параметра $\tau_{x y}^{\max }$ на линиях контакта полос, полосы и полуплоскости.

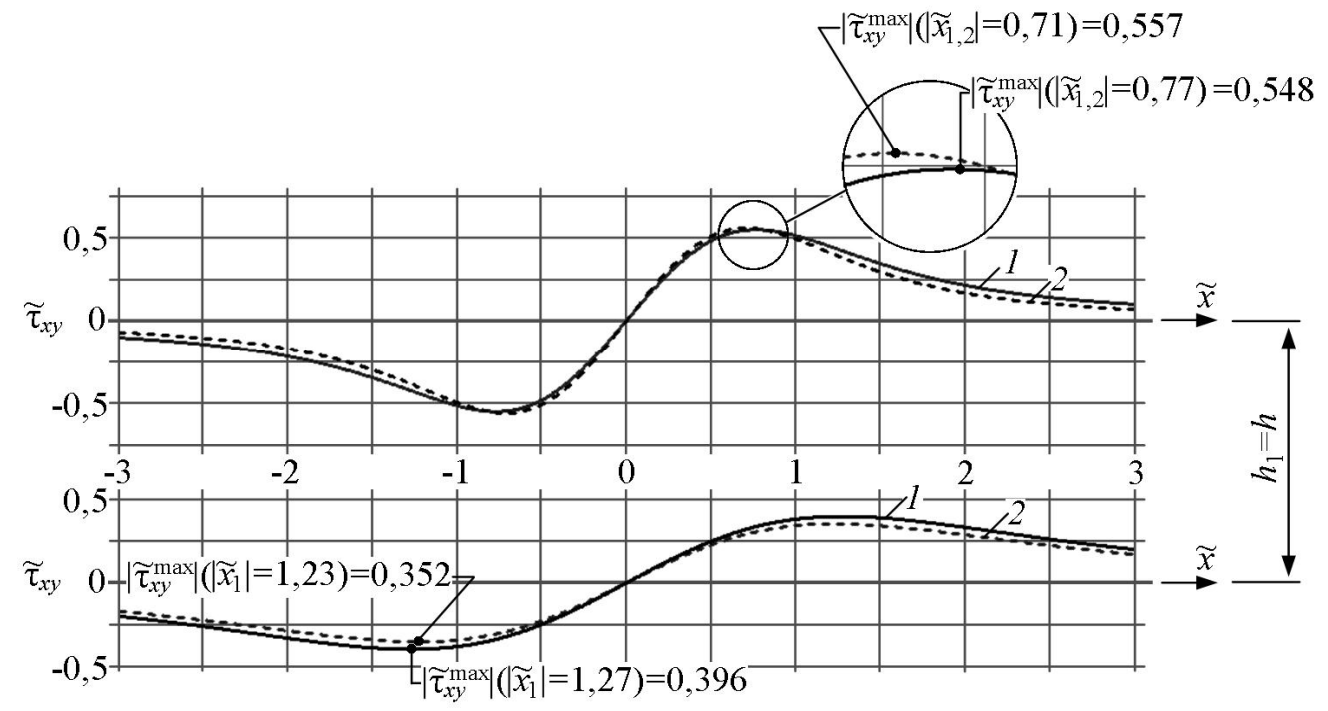

Рис.5. Изменение параметра $\tilde{\tau}_{x y}$ на линии контакта слоев

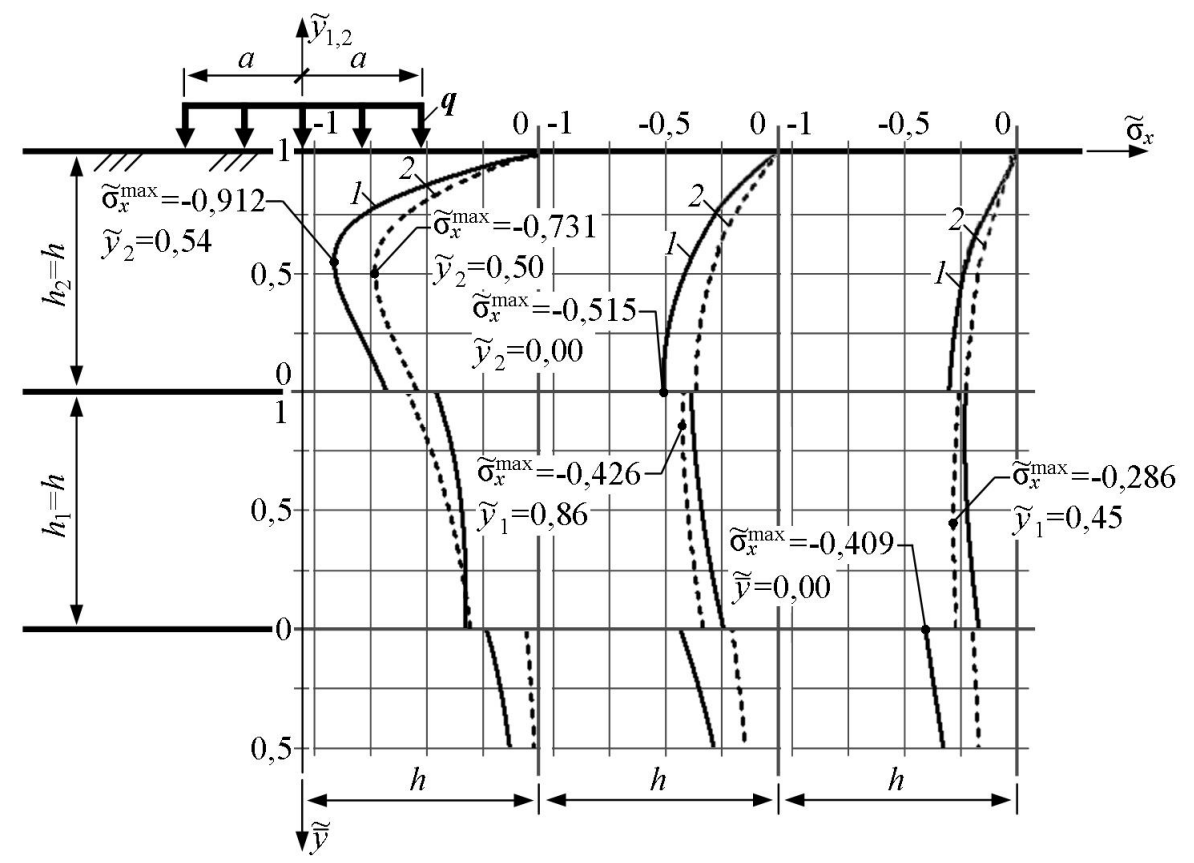

Рис.6. Эпюры распределения параметра $\widetilde{\sigma}_{x}$ по высоте основания

На рис. 6 представлены эпюры распределения параметра $\widetilde{\sigma}_{x}$ для вертикальных сечений. На графиках в каждом сечении указано значение $\widetilde{\sigma}_{x}^{\max }$ и координата $\tilde{y}_{k}(\widetilde{\bar{y}})$, в которой оно возникает.

Исходя из уравнений, которые были использованы при проведении расчетов, нетрудно проанализировать характер напряженно-деформированного состояния для частных случаев: двухслойная анизотропная полоса при условии полного сцепления между слоями скреплена с жестким основанием; однослой- 
ная анизотропная полоса скреплена с жестким основанием; анизотропная полоса скреплена с анизотропной полуплоскостью; анизотропная полуплоскость. Путем перехода от анизотропного материала к изотропному [15] можно рассмотреть аналогичный класс задач, когда одни слои составлены из изотропного материала, другие - анизотропного.

(C) Кудрявцев С.Г., Булдакова Ю.М. 2017

Спи сок литературы $320 \mathrm{c}$.

1. Харр M.Е. Основы теоретической механики грунтов. - М.: Стройиздат, 1971. -

2. Смирнов А.В., МальшевА.А., Ю.А. Агалаков. Механика устойчивости и разрушений дорожных конструкций. - Омск: СибАДИ, 1997. - 91 с.

3. Шехтер О.Я. Расчет бесконечной фундаментальной плиты, лежащей на упругом основании конечной и бесконечной мощности и нагруженной сосредоточенной силой // Сборник трудов научно-исследовательского сектора треста глубинных работ. - М.: Госстройиздат, 1939. - С. 133-139.

4. Pannonopm P.M. Задача Буссинеска для слоистого упругого полупространства// Труды Ленинградского политехнического института. - 1948. - № 5. - С. 3-18.

5. Коган Б.И. Напряжения и деформации многослойных покрытий // Труды ХАДИ. - 1953. - Вып.14. - С. 33- 46.

6. Кулагина М.Ф., Иванова В.И. Первая основная задача теории упругости для области, состоящей из полосы и полуплоскости // Вестник Самарского государственного технического университета. Серия: Физико-математические науки. -2003. - № 19. C. $89-96$.

7. Потележко В.П. Задача Фламана для двухслойной полуплоскости // Механика и физика процессов на поверхности и в контакте твердых тел, деталей технологического и энергетического оборудования. - 2005. - № 1. - С. 29-33.

8. Голеско В.A. Определение радиальных напряжений и вертикальных перемещений в характерных точках трехслойного полупространства по аппроксимирующим зависимостям // Вестник Харьковского национального автомобильно-дорожного университета. - 2005. - № 30. - С. 33-35.

9. Торская Е.В., Лушников Н.А., Лушников П.А. Анализ напряженно-деформированного состояния многослойных дорожных одежд // Трение и износ. -2008 . - Т.29, № 2. C. $204-210$.

10. Ширунов Г.Н. Метод начальных функций в модели упругого многослойного основания под действием нормальной локальной нагрузки // Инженерно-строительный журнал. - 2015. - №1(53). - С. 91-96.

11. Pan E. (1989) Static response of transversely isotropic and layered half-space to general surface loads . Phys. Earth Planet Inter. Vol. 54. P. 353-363.

12. Бугров А.К., Голубев А.И. Анизотропные грунты и основания сооружений. СПб: Недра, 1993. - 245 с.

13. Круподеров А.В. Фундаментальные решения для многослойных трансверсально изотропных оснований // Известия ТулГУ. Науки о Земле. - 2011. - №1. - С. 137-146.

14. Fabrikant V.I. (2013) Tangential contact problems for several transversely isotropic elastic layers bonded to an elastic foundation. Journal of Engineering Mathematics. Vol. 81. Issue 1. P. 93-126.

15. Кудрявиев С.Г., Булдакова Ю.М. Напряженное и деформированное состояние двухслойного анизотропного основания // Строительная механика инженерных конструкций и сооружений. - 2015. - № 5. - С. 9-20.

16. Кудрявиев С.Г., Булдакова Ю.М. Взаимодействие анизотропной полосы и жесткого основания // Строительная механика инженерных конструкций и сооружений. 2012. - № 4. - С. 29-35.

Поступила в редакиию 2 июля 2017 г. Прошла реиензирование 8 сентября 2017 г. Принята к публикации 18 октября 2017 г. 


\section{Об авторах:}

КУДРЯВЦЕВ СЕРГЕЙ ГЕННАДЬЕВИЧ - кандидат технических наук, доцент. В 1974 окончил механический факультет Марийского политехнического института им. М. Горького и по распределению оставлен для работы на кафедре сопротивления материалов и теоретической механики в должсности ассистента. 1978-1980 2.2. - аспирант кафедры сопротивления материалов и теории упругости Московского инженерно-строительного института им. В.В. Куйбышева (научный руководитель - профессор, д.т.н. Лукаш П.А.). В 1987 г. защитил кандидатскую диссертацию по специальности 05.23.17 Строительная механика. В 1991 году избран на должность доиента кафедры сопротивления материалов. Почетный работник высшего профессионального образования Российской Федерации, заслуженный работник образования республики Марий Эл. Область научных интересов: линейные и нелинейные задачи теории упругости.

Почтовый адрес: 424000, Республика Марий Эл, г. Йошкар-Ола, пл. Ленина, дом 3, кафедра сопротивления материалов и прикладной механики ФГБОУ ВО «ПГТУ».

БУЛДАКОВА ЮЛИЯ МИХАЙЛОВНА - старший преподаватель кафедры сопротивления материалов и прикладной механики ФГБОУ ВО «ПГТУ». В 2010 году окончила строительный факультет в ГОУ ВПО "Марийский государственный технический университет" с присуждением квалификации инженер. С 2010 по 2013 год аспирант кафедры сопротивления материалов и прикладной механики ФГБОУ ВПО «ПГТУ». Работает на кафедре с 2014 года. Область научных интересов: линейные задачи теории упругости анизотропных тел.

Почтовый адрес: 424000, Республика Марий Эл, г. Йошкар-Ола, пл. Ленина, дом 3, кафедра сопротивления материалов и прикладной механики ФГБОУ ВО «ПГТУ».

\section{Для цитирования:}

Кудрявцев С.Г., Булдакова Ю.М. Анализ напряженного состояния трехслойного анизотропного основания // Строительная механика инженерных конструкций и сооружений. - 2017. - № 6. - C. 16-24. Doi: 10.22363/1815-5235-2017-6-16-24.

\section{References}

1. Harr, M.E. (1971). Foundations of Theoretical Soil Mechanics. M.: Stroyizdat, 320 p. (In Russ.)

2. Smirnov, A.V., Malyshev, A.A., Agalakov, Y.A. (1997). Mechanics of the Stability and Destruction of Road Constructions. Omsk: SibADI. 91 p. (In Russ.)

3. Shekhter, O.Y. (1939). Calculation of an infinite fundamental plate lying on an elastic base of finite and infinite power and loaded with a concentrated force. Collected Works of the Research Sector of the Trust of Deep Works. M.: Gosstroiizdat. 133-139. (In Russ.)

4. Rappoport, R.M. (1948). The Boussinesq problem for a layered elastic half-space. Proceedings of the Leningrad Polytechnic Institute No 5. 3-18. (In Russ.)

5. Kogan, B.I. (1953). Stresses and deformations of multilayer coatings. Proceedings of HADI. Issue 14. 33-46. (In Russ.)

6. Kulagina, M.F., Ivanova, V.I. (2003). The first fundamental problem of the theory of elasticity for a domain consisting of a strip and a half-plane. Journal of Samara State Technical University, Ser. Physical and Mathematical Sciences. No 19. 89-96. (In Russ.)

7. Potelezhko, V.P. (2005). The Flaman problem for a two-layer half-plane. Mechanics and Physics of Processes on the Surface and in the Contact of Solids, Parts of Technological and Power Equipment. No 1. 29-33. (In Russ.)

8. Golesko, V.A. (2005). Determination of radial stresses and vertical displacements at characteristic points of a three-layer half-space from approximating relationships. Bulletin of the Kharkov National Automobile and Highway University. No 30. 33-35. (In Russ.)

9. Torskaya, E.V., Lushnikov, N.A., Lushnikov, P.A. (2008). Analysis of the stress-strain state of multilayer pavements. Friction and Wear, Vol. 29, № 2. 204-210. (In Russ.)

10. Shirunov, G.N. (2015). Method of initial functions in model of compression linearly deformable layered foundation under normal local load. Magazine of Civil Engineering. №1(53). 91—96. (In Russ.)

11. Pan, E. (1989). Static response of transversely isotropic and layered half-space to general surface loads. Phys. Earth Planet Inter. Vol. 54. 353-363.

12. Bugrov, A.K., Golubev, A.I. (1993). Anisotropic Soils and Foundations of Structures. SPb.: Nedra, 245 p. (In Russ.)

13. Krupoderov, A.V. (2011). Fundamental solutions for multilayer transversal-isotropic bases. Izvestiya of TSU. Sciences of Earth. No 1. 137-146. (In Russ.) 
14. Fabrikant, V.I. (2013). Tangential contact problems for several transversely isotropic elastic layers bonded to an elastic foundation. Journal of Engineering Mathematics, Vol. 81, Iss. 1. 93-126.

15. Kudryavtsev, S.G., Buldakova, J.M. (2015). Stress-strain state of two-layered anisotropic foundation. Structural Mechanics of Engineering Constructions and Buildings. No 5. 9-20. (In Russ.)

16. Kudryavtsev, S.G., Buldakova, J.M. (2012). Interaction of anisotropic band and rigid base. Structural Mechanics of Engineering Constructions and Buildings. No 4. 29-35. (In Russ.)

\title{
ANALYSIS OF THE STRESS STATE OF THREE-LAYERED ANISOTROPIC FOUNDATION
}

\author{
S.G. KUDRYAVTSEV, J.M. BULDAKOVA \\ Federal State Budgetary Educational Institution of Higher Professional Education \\ "Volga State University of Technology"
}

The results of calculations based on the equations of the plane problem of the theory of elasticity for determining stresses in a three-layer anisotropic base under the action of a static normal surface load are presented. It is considered a variant of the complete cohesion between the layers.

The stress functions in each layer are written through the normal and tangential forces arising on the line of contact between the strip and the half-plane. The contact forces between the strip and the half-plane are determined from the system of two equations and conditions on the surface of the base. As a result, the stress functions in each layer are written through the surface load in the form of improper integrals.

An analysis of the stress state is carried out according to the model of a three-layer isotropic base, depending on the elastic characteristics of the material of the layers. The influence of anisotropy of the material of layers in a three-layer soil mass is shown.

Key words: stress, anisotropy, elasticity, strip, half-plane.

Article history: Received: July 2, 2017. Revised: September 8, 2017. Accepted: October 18, 2017.

\section{About the authors:}

KUDRYAVTSEV SERGEY GENNADIEVICH, candidate of technical sciences, docent. In 1974, he graduated from the mechanical faculty of the Mari Polytechnic Institute named after M. Gorky and the distribution of the strength of materials and theoretical mechanics in the post of assistant was left for work at the department. 1978-1980: postgraduate of the department of strength of materials and the theory of elasticity of the Moscow Institute of Civil Engineering named after V.V. Kuibyshev (scientific leader-Professor, doctor of technical Sciences Lucas P.A.). In 1987, he defended his thesis on specialty 05.23.17" Structural Mechanics". In 1991, he was elected to the post of docent of strength of materials. Honored Worker of Higher Professional Education of the Russian Federation, Honored Worker of Education of the Republic of Mari El. Research interests: linear and nonlinear problems in the theory of elasticity.

Mailing address: 424000, the Republic of Mari El, Yoshkar-Ola, Lenin Sq., b. 3, the Department of materials resistance and applied mechanics, Federal State Budgetary Educational Institution of Higher Professional Education "Volga State University of Technology".

BULDAKOVA JULIA MIKHAILOVNA, senior lecturer of chair of materials resistance and applied mechanics of the Volga State University of Technology. In 2010, she graduated from the faculty of civil engineering of Mari State Technical University with the award of the qualification the engineer. From 2010 to 2013, she was postgraduate student of chair of materials resistance and applied mechanics of Volga Tech. She is working at the Department since 2014. Research interests: linear theory of elasticity of anisotropic bodies.

Mailing address: 424000, the Republic of Mari El, Yoshkar-Ola, Lenin Sq., b. 3, the Department of materials resistance and applied mechanics, Federal State Budgetary Educational Institution of Higher Professional Education "Volga State University of Technology".

\section{For citation:}

Kudryavtsev S.G., Buldakova J.M. (2017) Analysis of the stress state of three-layered anisotropic foundation. Structural Mechanics of Engineering Constructions and Buildings. No 5. 16-24. Doi: 10.22363/1815-5235-2017-6-16-24. (In Russ.) 